\title{
Estudio de la Microbiota del Proceso de Producción de Almidón Agrio de Yuca
}

\author{
Laura N. Chiquiza-Montaño(1)*, Olga I. Montoya ${ }^{(1)}$, Claudia Restrepo ${ }^{(2)}$ y Fernando Orozco-Sánchez ${ }^{(1)}$ \\ (1) Facultad de Ciencias, Universidad Nacional de Colombia, Sede Medellín, Calle 59A 63-20, Medellín- \\ Colombia. (e-mail: Inchiqui@unal.edu.co) \\ (2) Fundación Intal, Carrera 50G \# 12S - 91, Itagüi, Colombia.
}

*Autor a quien debe ser dirigida la correspondencia.

Recibido Dic. 21, 2015; Aceptado Feb. 22, 2016; Versión final Abr. 22, 2016, Publicado Oct. 2016

\section{Resumen}

Se recolectaron muestras líquidas de diferentes etapas de producción de almidón agrio de una industria local con el fin de evaluar y caracterizar la microbiota presente en el proceso. Las muestras se inocularon en diferentes medios de cultivo y se cuantificó la carga microbiana de cada una de ellas. Posteriormente, se obtuvieron 29 aislados de levaduras y 47 de bacterias que fueron caracterizados macro y microscópicamente. Finalmente, se evaluó la presencia de actividad amilolítica de los aislados y se identificaron 12 microorganismos, entre ellos Bacillus amyloliquefaciens, Bacillus vallismortis, Bacillus cereus, Leuconostoc mesenteroides y Saccharomyces cereviseae. La investigación muestra la variedad de microorganismos que se ven involucrados en el proceso fermentativo del almidón de yuca y sirve como base para futuras investigaciones que busquen mejorar el proceso de producción de almidones modificados por vía biotecnológica.

Palabras clave: almidón de yuca; almidón agrio; bacterias; levaduras; actividad amilolítica

\section{Microbiota Study of the Production Process of Sour Cassava Starch}

\begin{abstract}
Liquid samples were collected from different stages of sour starch production from a local industry with the aim of evaluating and microbiologically characterizing this process. Samples were taken and inoculated in different culture media. The microbiota of each sample was quantified and subsequently, 29 yeast strains and 47 bacterial strains were isolated, purified and characterized macro and microscopically. Finally, amilolitic activity was evaluated and twelve microorganisms were identified, including Bacillus amyloliquefaciens, Bacillus vallismortis, Bacillus cereus, Leuconostoc mesenteroides and Saccharomyces cereviseae. This research shows the variety of microorganisms that are involved in this fermentation process and also serves as a preliminary analysis for future research aiming to improve the production process of fermented starch.
\end{abstract}

Keywords: cassava starch; sour starch; bacteria; yeasts; amilolitic activity 


\section{INTRODUCCIÓN}

El almidón es un polisacárido de reserva energética de los vegetales (Witczak et al., 2015), es extraído de la yuca y utilizado de forma nativa (sin modificaciones) y con modificaciones físicas, químicas, enzimáticas o combinadas en las industrias de textiles, papel, adhesivos, farmacéuticas y de alimentos. En Colombia, dicho almidón es extraído de las raíces y se le llama almidón dulce; según su uso final, en algunos casos sufre de un proceso de fermentación natural dando como resultado el almidón agrio (Vargas Aguilar et al., 2012). Este último se obtiene de forma artesanal en establecimientos rurales agroindustriales llamados rallanderías cuyo producto principal es el almidón hidrolizado, conocido como almidón agrio utilizado en la industria panadera (Velasco, 2008). En las rallanderías se toman las raíces frescas, se lavan y con unas aspas giratorias por fricción entre ellas mismas, se remueven las cáscaras. Posteriormente, se quitan las puntas y las raíces se llevan al rallador, en el que se libera el almidón separándose los gránulos de las fibras. De esta última etapa depende el rendimiento en la obtención de almidón, porque un rallado muy fino provoca daño físico en los gránulos, una sedimentación más lenta y un rápido deterioro enzimático. Finalmente, la extracción termina en unos canales en los que el almidón se sedimenta y después de retirar la fase acuosa, se seca y se obtiene el almidón nativo (Alarcón y Dufuor, 1998). Sin embargo, la etapa de extracción de almidón puede verse afectada por varios factores artificiales que influyen en el rendimiento del almidón, incluyendo las condiciones de secado de las materias primas (Olomo y Ajibola, 2003), el tiempo de la cosecha y el almacenamiento de la raíz, debido a que éste disminuye el contenido de almidón (Benesi et al., 2008; Benesi et al., 2004). Todos estos factores pueden ser controlados y con una cuidadosa selección de los parámetros, puede maximizarse el rendimiento de la obtención de almidón.

Este tipo de almidón es utilizado como aditivo en diferentes industrias o para su posterior fermentación y obtención de almidón agrio, el cual se lleva en tanques de fermentación y se cubre por una capa del líquido sobrenadante de la sedimentación en periodos que van 20 a 90 días (Alarcón y Dufuor, 1998). Este almidón es usado en la fabricación de productos de panadería tradicionales y se obtiene en procesos cuyos parámetros no están bien definidos, por lo cual existe variación en la calidad del producto final (Acosta, 2006), al darse de manera natural y bajo condiciones ambientales, no está controlado, ni estandarizado, y no se aplican los principios de Buenas Prácticas de Manufactura (BPM) (Cadena et al., 2006). Sin embargo, esta etapa depende de la presencia de microorganismos amilolíticos que degradan parcialmente el almidón, produciéndose azúcares simples que constituyen a su vez, el sustrato de microorganismos productores de ácidos orgánicos, como láctico, propiónico, acético y butírico (Nunes y Cereda, 1994). Este último paso implica la presencia de Bacterias Ácido Lácticas (BAL) de los géneros Streptococcus, Lactococcus y Lactobacillus, además, de levaduras del género Saccharomyces (Cárdenas y Buckle, 1980; Figueroa et al., 1995; George et al., 1995), siendo las BAL más abundantes que las levaduras (Parada et al., 1996).

Cereda (1975) concluyó que este proceso fermentativo se desarrolla en tres fases; en la primera, se desarrolla una microbiota poco exigente, que consiste principalmente en los grupos coliformes y mesófilos aerobios. En la segunda, intervienen microorganismos más exigentes identificados como productores de ácidos orgánicos, muchos de los cuales pertenecen a las BAL, y se caracterizan por ser microaerófilos o anaerobios; en cambio, en la tercera fase predominan las levaduras saprofitas. De las anteriores etapas, la acidificación láctica junto con la energía suficiente de los rayos UV, dan como resultado la depolimerización parcial de las moléculas del almidón, lo cual produce pequeños fragmentos lineales. Estos últimos, junto con algunos radicales, ayudan a la reticulación de las moléculas de almidón restantes, formando una red tridimensional que se traduce en las propiedades de expansión del almidón agrio durante la cocción (Alarcón y Dufuor, 1998).

Debido a las condiciones ambientales, a la falta de estandarización y a la diversidad microbiana en las que se dan los procesos de extracción y fermentación del almidón de yuca, se puede dar una mayor variabilidad en la obtención de dicho almidón. Por lo tanto, el objetivo de esta investigación fue identificar y seleccionar algunos microorganismos con actividad amilolítica que intervienen en cada una de las etapas de la producción de almidón agrio.

\section{MATERIALES Y MÉTODOS}

Se describe la obtención de muestras microbianas, la siembra y aislamiento de microorganismos, la identificación de los aislados y la determinación de capacidad amilolítica en lo que sigue.

\section{Obtención de muestras microbianas}

Se recolectaron muestras de varias etapas del proceso de extracción y fermentación de almidón, de una rallandería localizada en el municipio de Sampués, departamento de Sucre, Colombia; figura 1. Se tomaron un total de 40 muestras al azar, sin repeticiones; 22 líquidas, cada una aproximadamente de $200 \mathrm{~mL}$ y 18 
sólidas cada una de $500 \mathrm{~g}$ peso húmedo, posteriormente fueron llevadas a bolsas estériles Nasco Whirlpak, divididas en muestras del proceso de extracción y proceso de fermentación; cinco muestras líquidas y una sólida provenientes del primer proceso: desde los flujos de entrada de agua y entrada a la sedimentación, flujos de salida del rallador y salida del colador y agua que queda como sobrenadante en la sedimentación (agua de fermentación). La muestra sólida corresponde al almidón extraído. Además, del segundo proceso (fermentación), se tomaron 17 muestras sólidas y 17 líquidas procedentes de cuatro tipos de tanques. En el lugar, a cada una de las muestras líquidas se les midió pH y temperatura. Las muestras fueron transportadas en contenedores de plástico estériles refrigerados al laboratorio de Microbiología de Aguas y Alimentos de la Universidad Nacional de Colombia, sede Medellín y al laboratorio de microbiología de la fundación intal y almacenadas a $4 \pm 2{ }^{\circ} \mathrm{C}$ hasta el momento de los análisis.

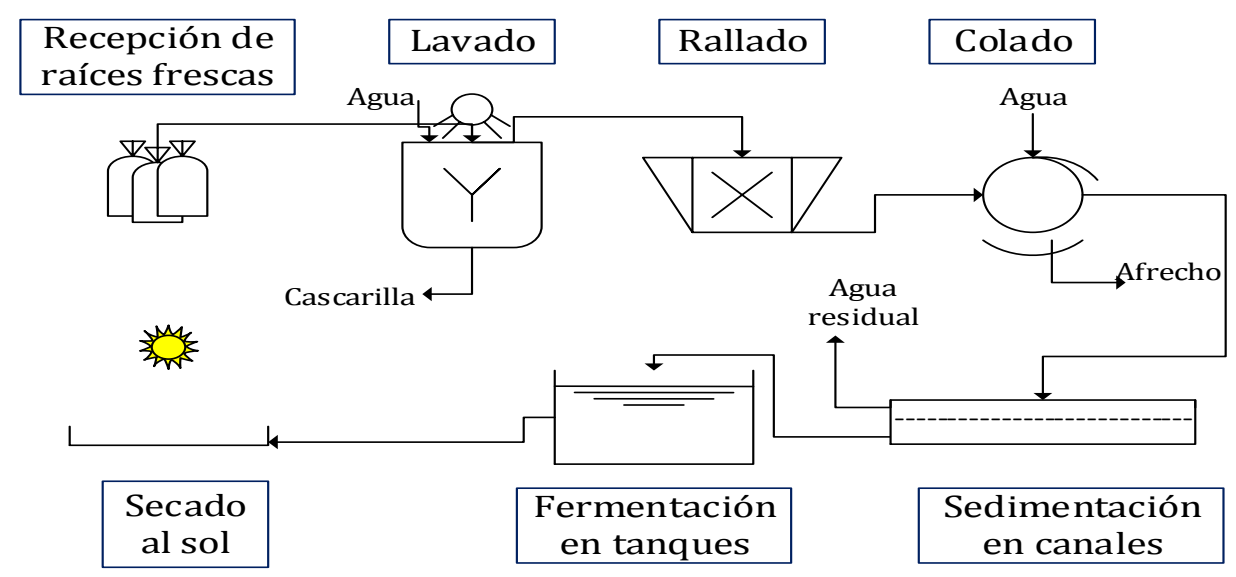

Fig. 1. Proceso de obtención de almidón agrio a partir de raíces de yuca.

\section{Siembra y aislamiento de microorganismos}

Una vez recolectadas y transportadas las muestras, se procedió a inocularlas en diferentes medios de cultivo sólidos: agar tripteína soya (TSA) para microorganismos viables a $37 \pm 2^{\circ} \mathrm{C}$ por 24 a 48 horas, diclorán rosa de bengala-cloranfenicol (DRBC) para hongos a $25 \pm 2^{\circ} \mathrm{C}$ de 3 a 5 días, Man Rogosa y Sharpe (MRS) para bacterias ácido lácticas a $37 \pm 2^{\circ} \mathrm{C}$ por 72 horas en cámaras con anaerocult y agar nutritivo enriquecido con $0,1 \% \mathrm{p} / \mathrm{v}$ de almidón soluble para microorganismos con actividad amilolítica a $37 \pm 2^{\circ} \mathrm{C}$ por 24 a 48 horas; por el método de agotamiento en superficie. Posteriormente, a cada uno de los cultivos microbianos se les realizó el recuento de Unidades Formadoras de Colonia (UFC)/mL mediante la técnica de recuento en placa en superficie. Para esto se inoculó $0.1 \mathrm{~mL}$ de las diluciones $10^{2}$ y $10^{4}$ en cajas Petri con los medios de cultivo estériles. Los conteos se realizaron en cuenta colonias. Además, se realizó la prueba de actividad amilolítica y se seleccionaron las cajas con mejor actividad, para así, aislarlas, purificarlas e identificarlas (Madigan et al. 2014).

El aislamiento de las colonias se realizó teniendo en cuenta las condiciones aerobias y aquellas que presentaron mejor actividad amilolítica. En el caso de los mohos y las levaduras se inocularon en agar extracto de levadura glucosa cloranfenicol (YGC) a $22 \pm 2^{\circ} \mathrm{C}$ durante 5 días y las bacterias en agar Nutritivo enriquecido con almidón a $37 \pm 2^{\circ} \mathrm{C}$ por 24 a 48 horas. Se procedió a la purificación de cada uno de los aislados, con base, a la caracterización macroscópica de la colonia con el uso del estereoscopio y microscopio y la morfología del microorganismo como tal, mediante el microscopio por medio de la tinción de Gram (Rojas-Triviño 2011).

\section{Identificación de los aislados.}

Al azar, dieciocho aislados fueron identificados por pruebas bioquímicas. Una vez caracterizados macroscópica y microscópicamente se llevaron a análisis al laboratorio de Tecnimicro, Medellín, donde se utilizó el equipo Vitek 2 Compact 60, versión instalada Systems 06.01 de BioMérieux, que consta de una serie de tarjetas para levaduras (YST), bacilos formadores de esporas Gram positivos (BCL), bacilos Gram negativos (GN), cocos y bacilos no formadores de esporas Gram positivos (GP) impregnadas con reactivos colorimétricos, los cuales se inocularon con la suspensión del cultivo puro. El perfil se interpretó de manera automática. Cada una de las tarjetas cuenta con 64 pozos y se midieron las actividades enzimáticas como consecuencia de la producción de metabolitos que cambian el pH hacia ácido o alcalino y son detectados por los indicadores de $\mathrm{pH}$ presentes en cada sustrato. Finalmente, el equipo arrojó una probabilidad de 
identificación del microorganismo según la comparación que realiza con su base de datos que da una precisión de 93\% para GP, 94\% para GN, 87\% para BCL y 84\% para YST. (BioMérieux 2015).

\section{Determinación de capacidad amilolítica}

A cada aislamiento puro, se le determinó la actividad amilolítica, inoculando los microorganismos en cajas de Petri con Agar Nutritivo enriquecido con $0.1 \% \mathrm{p} / \mathrm{v}$ de almidón soluble, incubadas a $37^{\circ} \mathrm{C} \pm 2^{\circ} \mathrm{C}$ durante 24 a 48 horas y $25^{\circ} \mathrm{C} \pm 2^{\circ} \mathrm{C}$ por 120 horas, bacterias y levaduras respectivamente. Posteriormente, se adicionó al cultivo microbiano una cantidad de solución de yodo (KI-5,0\% p/v y I 2-0,5\% p/v). La presencia de un complejo helicoidal entre amilosa y yodo, da lugar al típico azul profundo de dispersiones de almidón teñidos con yodo (McGrance, Cornell, y Rix 1998). La formación de halos claros o transparentes mayores a $1 \mathrm{~mm}$ de radio alrededor del crecimiento demostraron la actividad amilolítica; a mayor halo mayor actividad, por lo que la distancia de la zona desde el borde de la colonia hasta el límite externo, demuestran la capacidad del microorganismo de hidrolizar dicho sustrato (Madigan et al. 2014).

\section{RESULTADOS Y DISCUSIÓN}

Se presentan los resultados de la caracterización de las muestras recolectadas, de la caracterización microbiana y de la identificación de los aislados y la actividad amilolítica de los aislados

\section{Caracterización de las muestras recolectadas}

Se tomaron muestras de todo el proceso en la rallandería, desde la extracción hasta la fermentación del almidón, figuras 2 a 5, y datos de temperaturas y $\mathrm{pH}$. Para el segundo proceso, también se tuvo en cuenta el tiempo que llevaba el almidón en el mismo.

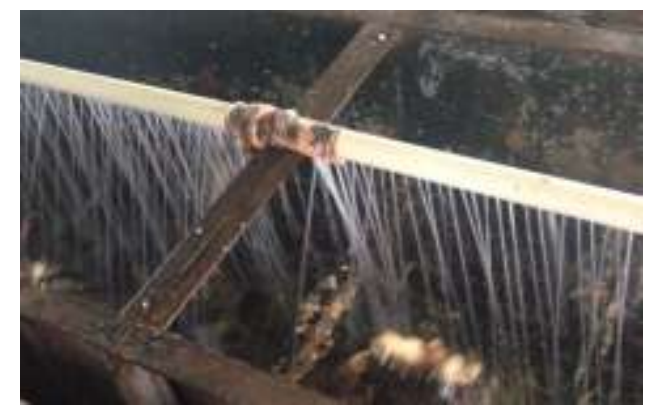

Fig. 2. Lavado de yuca.

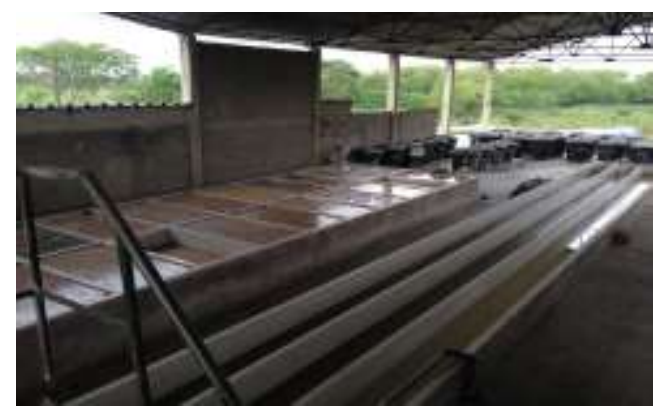

Fig. 3. Canales de sedimentación y tanques de fermentación

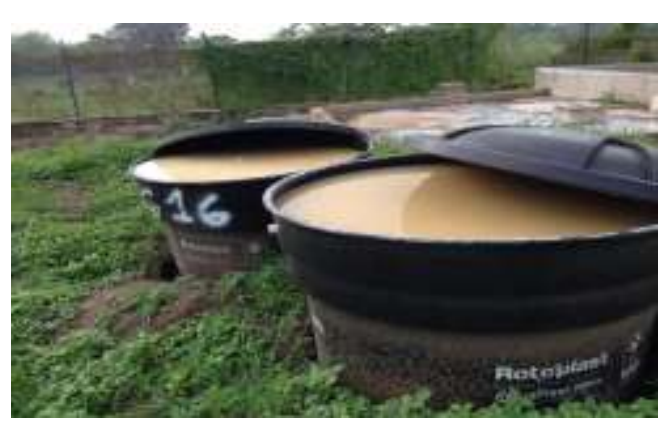

Fig. 4. Tanques de fermentación Rotoplast

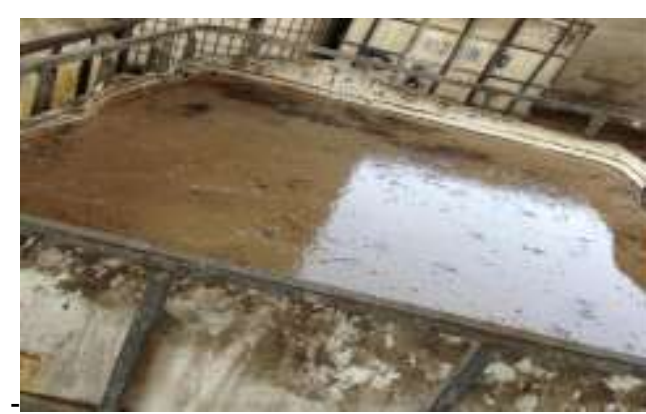

Fig. 5. Tanques de fermentación de plástico

La ralandería en la cual se realizó la recolección se encuentra ubicada en la periferia del municipio de Sampués, Sucre; en esta región se cultiva yuca, por lo que esta no sufre de largas distancias de transporte que afecte su procesamiento, cuenta con una temperatura promedio de $32{ }^{\circ} \mathrm{C}$ y una humedad relativa de $74 \%$. Se registraron temperaturas desde 25 hasta $39.5^{\circ} \mathrm{C}$ en los procesos (extracción y fermentación), las cuales no presentaron un cambio drástico, y corresponden a la temperatura ambiental del lugar y la hora de la toma de muestra (cerca al mediodía), a excepción de la temperatura del agua de entrada, la cual no se encuentra expuesta a las mismas condiciones ambientales. Estas temperaturas favorecen el crecimiento y metabolismo de microorganismos mesófilos.

Por el contrario, en el proceso de fermentación, llevado a cabo en diferentes tipos de tanques; de cemento (TC), de plástico blanco cúbico (TP), de plástico negro cilíndrico de 1000 L (TR) y de plástico negro cilíndrico de 2000 L (TRG) y en el líquido para la fermentación (AS) se presentó una disminución de pH, que 
alcanzó valores de 3.3 que difieren del valor promedio de la extracción $(6.6 \pm 1.4)$ posiblemente por la producción de ácidos orgánicos de los microorganismos asociados al proceso (Marcon et al. 2006). El cambio que muestra el pH no presenta una relación directa con el número de días de la fermentación, esto puede explicarse por la degradación de almidón que genera moléculas de glucosa precursoras de la síntesis de ácidos. Como es un sistema expuesto al ambiente, la superficie es susceptible a contaminaciones que alteren los datos al interior de éste. Estos valores de $\mathrm{pH}$ registrados corresponden a los reportados por Cadena (2006) que se encuentran en un intervalo de $\mathrm{pH}$ de 3 a 6 , quienes tampoco observaron una tendencia de estos datos en comparación con el número de días de fermentación, ni con las características tecnológicas que presentan las diferentes rallanderías que fueron evaluadas en ese trabajo.

\section{Caracterización microbiana}

Durante el proceso de extracción, los recuentos de mohos y levaduras oscilaron entre $2 \times 10^{2}$ y $1.5 \times 10^{4}$ $\mathrm{UFC} / \mathrm{mL}$, y las BAL entre 55 y $21 \times 10^{5} \mathrm{UFC} / \mathrm{mL}$. Por otra parte, en el proceso de fermentación para la obtención de almidón agrio, los mohos y levaduras se cuantificaron en un intervalo de $>10$ hasta $28 \times 10^{4}$ $\mathrm{UFC} / \mathrm{mL}$ y las BAL de $14 \times 10^{2}$ hasta $15 \times 10^{8} \mathrm{UFC} / \mathrm{mL}$. No todas las muestras dieron positivo a la prueba de hidrólisis realizada con lugol Tabla 1. Figueroa (Figueroa, Davila, y Pourquié 1995) y Ampe (Ampe, Sirvent, y Zakhia 2001) observaron en la fermentación natural de yuca, que los grupos microbianos predominantes son BAL (108 a $10^{9}$ UFC log/g) y levaduras (10 a $10^{4} \log$ UFC/g).

Tabla 1. Carga microbiana muestras proceso producción almidón dulce y agrio

\begin{tabular}{|l|l|l|l|l|}
\hline Código & Act. Amilolítica & Mohos (UFC/mL) & Levaduras (UFC/mL) & BAL (UFC/mL) x10 \\
\hline Agua de entrada & + & 960 & 1600 & 0,55 \\
\hline Agua salida del rallador & + & 3700 & 15000 & 2100 \\
\hline Agua salida del colador & + & 230 & 200 & 190 \\
\hline Agua salida al sedimentador & - & 650 & 4100 & 5 \\
\hline Agua de fermentación & + & 820 & 4000 & 420000 \\
\hline TC 5 & + & 10 & 100 & 500 \\
\hline TC 7 & + & 10 & 200 & 1300 \\
\hline TC 8 & + & 100 & 56000 & 3,5 \\
\hline TC 12 & + & 20 & 300 & 5,4 \\
\hline TC 13 & + & $<10$ & 25000 & 24 \\
\hline TC 16 & + & $<10$ & 55000 & 15 \\
\hline TC 17 & + & $<10$ & 280000 & 1400 \\
\hline TC 22 & + & 30 & 60 & 350 \\
\hline TR 4 & + & 7500 & 8100 & 500 \\
\hline TR 9 & - & 20 & 4600 & 51 \\
\hline TR 15 & - & $<10$ & $<10$ & 320 \\
\hline TRG 1 & - & 100 & 80 & 1300 \\
\hline TRG 9 & + & $<10$ & $<10$ & 3400 \\
\hline TRG 12 & - & $<10$ & $<10$ & 600 \\
\hline TRG 15 & + & $<10$ & $<10$ & 1,4 \\
\hline TP 2 & + & - & 150 & 520 \\
\hline TP 8 & -200 & 84000 & 1500000 \\
\hline
\end{tabular}

Aunque, el recuento microbiano no arrojó una tendencia clara de la presencia o cantidad de los mismos en las etapas del proceso extracción y fermentación, ni en los diferentes días de fermentación, es posible observar una menor cantidad de mohos, en algunos casos $<10$ y un aumento de BAL que alcanza $1.5 \times 10^{9}$ UFC/mL en la etapa fermentativa, lo cual concuerda con la disminución del pH en la misma. Asimismo, los aislados de mejor actividad amilolítica provenían de las muestras que correspondían a 3 días diferentes de fermentación, 2 de salidas del proceso y el agua que queda luego de la sedimentación.

Se purificaron y aislaron un total de 29 colonias de levaduras diferentes y 47 colonias bacterianas diferentes (datos no mostrados), la mayoría bacilos esporulados y como era de esperarse muchas de estas 
presentaban características muy semejantes entre ellas. Aunque, la caracterización macro y microscópica es útil para diferenciar, no garantiza que no se trata del mismo microorganismo, por lo tanto, es necesario hacerle un perfil bioquímico amplio o una caracterización molecular. Dadas las condiciones de $\mathrm{pH}$, un número significativo de cepas bacterianas es encontrado en el agua inicial de la fermentación

\section{Identificación de los aislados}

Mediante identificación bioquímica y posterior comparación en las bases de datos se encontraron 12 microorganismos diferentes.

Saccharomyces cerevisiae. Encontrada en el agua de fermentación. El crecimiento obtenido presentó características macroscópicas de colonias de textura cremosa, forma circular, borde entero y superficie lisa y características microscópicas de levadura. Este microorganismo es una levadura facultativa, cuando crece en medios anerobios ricos en glucosa, maltosa, fructosa o sacarosa, produce etanol y $\mathrm{CO}_{2}$. Se utilizó la tarjeta YGC para su identificación que obtuvo un 95\% de probabilidad. Detalles bioquímicos: LysA (-), IMLTa $(-)$, LeuA (+), ARG (-), ERYa (-), GLYLa(-), TyrA (-), BNAG (-), ARBa (-), AMYa (-), dGALa (+), GENa (-), dGLUa (+), LACa (-), MadGa (-), dCELa (-), GGT (-), dMALa (-), dRAFa (+), NAGA1 (-), dMNEa (+), dMELa $(-)$, dMLZa (-), ISBEa (-), IRHAa (-), XLTa (-), dSORa (-), SACa (+), URE (-), AGLU (-), dTURa (-), dTREa $(+)$, NO3a (-), IARA (-), dGATa (+), ESC (-), IGLTa (-), dXYLa (-), LATa (-), ACEa (+), CITa (-), GRTas (-), IPROa (-), 2KGa (-), NAGa (-), dGNTa (-).

Leuconostoc mesenteroides ssp. Destranicum. Encontrada en el agua de fermentación. Estas colonias presentaron unas características de crecimiento macroscópicas de textura cremosa, forma circular y borde entero, y microscópica de bacilos Gram positivos. Estas bacterias producen dextranos a partir de glucosa, es anaerobia facultativa y se encuentra presente en vegetales. Se obtuvo un $88 \%$ de probabilidad utilizando la tarjeta GP. Detalles bioquímicos: AMY (+), PIPLC (-), dXYL (-), ADH1 (-), BGAL (+), AGLU (+), APPA (-), CDEX (-), AspA (-), BGAR (-), AMAN (-), PHOS (-), LeuA (-), ProA (-), BGURr (-), AGAL (+), PyrA (-), BGUR $(-)$, AlaA (-), TyrA (-), dSOR (-), URE (-), POLYB (+), dGAL (-), dRIB (-), ILATk (-), LAC (+), NAG (-), dMAL $(+)$, BACl (+), NOVO (+), NC6.5 (-), dMAN (-), dMNE (+), MBdG (+), PUL (-), dRAF (-), O129R (+), SAL (+), SAC (+), dTRE (+), ADH2s (-), OPTO (+).

En uno de los tanques de fermentación de cemento (1) y en el agua de fermentación se encontró Bacillus amyloliquefaciens. Presentó características macroscópicas (borde irregular, planas y consistencia mucosa) y microscópicas de bacilos Gram positivos esporulados. Este bacilo es aerobio, productor de $\alpha$-amilasa y se encuentra fácilmente en el suelo. En los dos casos fue usada la tarjeta BCL y se logró un $88 \%$ de probabilidad de la muestra provenente del tanque de fermentación y un $94 \%$ de probabilidad del agua de fermentación. Detalles bioquímicos de la muestra del tanque de fermentación: BXYL (+), LysA (-), AspA (-), LeuA (+), PheA (+), ProA (-), BGAL (+), PyrA (+), AGAL (+),AlaA (-), TyrA (+), BNAG (-), APPA (+), CDEX $(+)$, dGAL (-), GLYG (-), INO (+), MdG (+), ELLM (-), MdX (-), AMAN (-), MTE (+), GlyA (+), dMAN (+), dMNE $(-)$, dMLZ (-), NAG (-), PLE (-), IRHA (-), BGLU (+), BMAN (-), PHC (-), PVATE (-), AGLU (+), dTAG (-), dTRE (+), INU (-), dGLU (+), dRIB (-), PSCNa (-), NaCl 6.5\% (+), KAN (-), OLD (-), ESC (+), TTZ (-), POLYB_R (-). Detalles bioquímicos en que difiere la muestra del agua de fermentación de la del tanque de fermentación: TyrA (-),GLYG (+), GlyA (-), dMNE (+), PLE (+), PVATE (+), INU (+), dGLU (+), dRIB (+), TTZ $(+)$, POLYB_R (+).

En la muestra del agua de salida del colador, se encontró Streptococcus mutans. El crecimiento obtenido presentó características macroscópicas (colonias de forma circular, textura cremosa y planas) y microscópica de bacilos Gram positivos cortos. Es un microorganismo anaerobio facultativo, acidófilo, metaboliza sacarosa para producir polisacáridos, frecuente en la cavidad bucal humana. Se obtuvo un $99 \%$ de probabilidad utilizando la tarjeta GP. Detalles bioquímicos: AMY (+), PIPLC (-), dXYL (-), ADH1 (-), BGAL $(-)$, AGLU (-), APPA (-), CDEX (-), AspA (-), BGAR (-), AMAN (-), PHOS (-), LeuA (+), ProA (-), BGURr (-), AGAL (-), PyrA (-), BGUR (-), AlaA (+), TyrA (-), dSOR (+), URE (-), POLYB (-), dGAL (-), dRIB (-), ILATk (-), LAC (-), NAG (+), dMAL (+), BACI (+), NOVO (+), NC6.5 (-), dMAN (+), dMNE (+), MBdG (+), PUL (-), dRAF $(-)$, O129R (-), SAL (+), SAC (+), dTRE (+), ADH2s (-), OPTO (+).

En el agua de salida del colador, el agua de fermentación y uno de los tanques de fermentación de cemento (1) se encontró Bacillus vallismortis. Se presentaron características macroscópicas (colonias de textura mucosa, planas, borde irregular y opacas) y microscópicas de bacilos Gram positivos esporulados. Éstas son bacterias aerobias, que hidrolizan almidón, y están presentes en suelo. Utilizando en los tres casos la tarjeta BCL se obtuvo un $85 \%$ de probabilidad en el agua de salida del colador y un $93 \%$ de probabilidad en el agua de fermentación y uno de los tanques de fermentación. Detalles bioquímicos: BXYL (+), LysA (+), AspA (-), LeuA (+), PheA (-), ProA (-), BGAL (+), PyrA (+), AGAL (+), AlaA (-), TyrA (-), BNAG (-), APPA (+), $\operatorname{CDEX}(+), \operatorname{dGAL}(-), \operatorname{GLYG}(+), \operatorname{INO}(-), \operatorname{MdG}(+), \operatorname{ELLM}(+), \operatorname{MdX}(-), \operatorname{AMAN}(-), \operatorname{MTE}(+), \operatorname{GlyA}(-)$, dMAN (+), 
dMNE (+), dMLZ (-), NAG (-), PLE (+), IRHA (-), BGLU (+), BMAN (-), PHC (-), PVATE (-), AGLU (+), dTAG $(-)$, dTRE (-), INU (-), dGLU (-), dRIB (-), PSCNa (-), NaCl 6.5\% (+), KAN (-), OLD (-), ESC (+), TTZ (-), POLYB_R (+). Detalles bioquímicos en que se diferencia la muestra del agua de fermentación de la muestra del sagua a la salida del colador: INO (+), PVATE (+), AGLU (-), dTRE (+), INU (+), dGLU (+), dRIB (+). Detalles bioquímicos en que es diferente la muestra del tanque de fermentación de la muestra del agua de la salida del colador: LeuA (-), INO (+), PVATE (+), AGLU (-), dTRE (+), INU (+), dGLU (+), dRIB (+),

En uno de los tanques de fermentación de plástico negro cilíndrico de 1000 L (1) se encontró Candida famata. El crecimiento de estas colonias presentó características macroscópicas (colonias de textura cremosa, consistencia blanda y superficie lisa) y microscópicas de levaduras. Éstas pueden fermentar glucosa, galactosa y sacarosa, es variable a almidón, asociada a la cavidad bucal humana. $85 \%$ de probabilidad utilizando la tarjeta YST. Detalles bioquímicos: LysA (-), IMLTa (+), LeuA (+), ARG (+), ERYa (), GLYLa(+), TyrA (-), BNAG (-), ARBa (+), AMYa (-), dGALa (+), GENa (-), dGLUa (+), LACa (+), MadGa $(+)$, dCELa (+), GGT (+), dMALa (+), dRAFa (+), NAGA1 (-), dMNEa (+), dMELa (+), dMLZa (+), ISBEa (-), IRHAa (-), XLTa (-), dSORa (+), SACa (+), URE (-), AGLU (+), dTURa (+), dTREa (+), NO3a (-), IARA (+), dGATa (+), ESC (+), IGLTa (+), dXYLa (+), LATa (+), ACEa (+), CITa (+), GRTas (+), IPROa (+), 2KGa (+), NAGa $(+)$, dGNTa (+).

En uno de los tanques de fermentación de cemento (2) se encontró Aeromonas salmonicida. Estas colonias presentaron características macroscópicas (colonias de borde entero, opacas y superficie lisa) y microscópicas de bacilos Gram negativos. Esta bacteria es anaerobia facultativa, presente en un medio acuoso y suelo. Patógena en peces. Usando la tarjeta GN se obtuvo un $92 \%$ probabilidad. Detalles bioquímicos: APPA (-), ADO (-), PyrA (+), IARL (-), dCEL (-), BGAL (-), H2S (-), BNAG (-), AGLTp (-), dGLU $(-)$, GGT (-), OFF (-), BGLU (-), dMAL (-), dMNE (-), BXYL (-), BAlap (-), ProA (+), LIP (-), PLE (-), TyrA (-), URE (-), dSOR (-), SAC (-), dTAG (-), dTRE (-), CIT (-), MNT (-), 5KG (-), ILATk (+), AGLU (-), SUCT (-), NAGA (-), AGAL (-), PHOS (-), GlyA (-), ODC (-), LDC (-), IHISa (-), CMT (-), BGUR (-), O129R (-), GGAA (), IMLTa (-), ELLM (+), ILATa (-).

En la salida del colador y un tanque de fermentación de cemento (3) se encontró Sphingomonas paucimobilis. El crecimiento obtenido presentó características macroscópicas (colonias de consistencia blanda, planas y superficie lisa) y microscópicas de cocobacilos Gram negativos. Este microorganismo es aerobio, patógeno, se encuentra fácilmente en un medio acuoso y suelo. En los dos casos se utilizó la tarjeta GN, para la muestra de la salida del colador se obtuvo un $93 \%$ de probabilidad y para el tanque de fermentación un $88 \%$ de probabilidad. Detalles bioquímicos de la muestra de la salida del colador: APPA $(+)$, ADO (-), PyrA (-), IARL (-), dCEL (-), BGAL (-), H2S (-), BNAG (-), AGLTp (+), dGLU (+), GGT (-), OFF (), BGLU (-), dMAL (-), dMNE (-), BXYL (-), BAlap (-), ProA (-), LIP (+), PLE (-), TyrA (+), URE (-), dSOR (-), SAC (-), dTAG (-), dTRE (-), CIT (-), MNT (-), 5KG (-), ILATk (+), AGLU (+), SUCT (+), NAGA (-), AGAL (-), PHOS (+), GlyA (+), ODC (-), LDC (-), IHISa (-), CMT (+), BGUR (-), O129R (-), GGAA (+), IMLTa (-), ELLM $(+)$, ILATa $(-)$. Detalles bioquímicos en que difiere la muestra del tanque de fermentación: APPA (-), dCEL $(+)$, BGAL (+), AGLTp (-), GGT (+), BGLU (+), dMAN (+), dMNE (+), BXYL (+), LIP (-), SAC (+), dTAG (+), dTRE (+), ILATk (-), AGLU (-), SUCT (-), PHOS (-), GlyA (-), CMT (-), GGAA (-), ELLM (-).

En uno de los tanques de fermentación de plástico negro cilíndrico de $2000 \mathrm{~L}$ se encontró Acinetobacter Iwolfii: El crecimiento obtenido presentó características macroscópicas (colonias de borde entero, opacas, consistencia blanda y superficie lisa) y microscópicas de bacilos largos Gram negativos. Es aerobio, patógeno, frecuente en el suelo. 92\% de probabilidad utilizando la tarjeta GN. Detalles bioquímicos: APPA $(-)$, ADO (-), PyrA (-), IARL (-), dCEL (-), BGAL (-), H2S (-), BNAG (-), AGLTp (-), dGLU (-), GGT (-), OFF (-), BGLU (-), dMAL (-), dMAN (-), dMNE (-), BXYL (-), BAlap (-), ProA (-), LIP (-), PLE (-), TyrA (+), URE (-), dSOR (-), SAC (-), dTAG (-), dTRE (-), CIT (+), MNT (-), 5KG (-), ILATk (+), AGLU (-), SUCT (+), NAGA (-), AGAL (-), PHOS (-), GlyA (+), ODC (-), LDC (-), IHISa (-), CMT (+), BGUR (-), O129R (-), GGAA (-), IMLTa (), ELLM (+), ILATa (-).

En uno de los tanques de fermentación de plástico negro cilíndrico de $1000 \mathrm{~L}$ (2) se encontró Cryptococcus laurentii. El crecimiento obtenido presentó características macroscópicas (colonias de borde entero, opacas, superficie lisa) y microcópicas de levaduras. Es un hongo patógeno, saprófito, no fermenta glucosa, sacarosa ni lactosa y asociado al suelo. Con la tarjeta YST arrojó un $87 \%$ de probabilidad. Detalles bioquímicos: LysA (+), IMLTa (+), LeuA (+), ARG (+), ERYa (-), GLYLa(+), TyrA (+), BNAG (-), ARBa (+), AMYa (-), dGALa (+), GENa (-), dGLUa (+), LACa (+), MadGa (-), dCELa (-), GGT (+), dMALa (+), dRAFa (+), NAGA1 (-), dMNEa (+), dMELa (+), dMLZa (-), ISBEa (+), IRHAa (-), XLTa (-), dSORa (-), SACa (+), URE (+), AGLU (+), dTURa (-), dTREa (+), NO3a (-), IARA (+), dGATa (+), ESC (+), IGLTa (+), dXYLa (+), LATa (+), ACEa (+), CITa (+), GRTas (+), IPROa (+), 2KGa (+), NAGa (+), dGNTa (+). 
En el agua de salida del colador, el agua de fermentación y uno de los tanques de fermentación de plástico se encontró Bacillus cereus. El crecimiento obtenido presentó características macroscópicas (crecimiento de colonias de consistencia mucosa, planas y opacas) y microscópicas de Bacilos Gram positivos esporulados. Es aerobio, causa intoxicación alimentaria y es frecuente en el suelo. En los tres casos se utilizó la tarjeta BCL, se obtuvo un $93 \%$ de probabilidad para la muestra del agua a la salida del colador, un $95 \%$ de probabilidad para la muestra del agua de fermentación y un $89 \%$ de probabilidad para la muetsra del tanque de fermentación. Detalles bioquímicos de la muestra de la salida del colador: BXYL (-), LysA (-), AspA (-), LeuA (-), PheA (+), ProA (-), BGAL (-), PyrA (-), AGAL (-),AlaA (-), TyrA (-), BNAG (+), APPA (-), CDEX (-), dGAL (-), GLYG (-), INO (-), MdG (-), ELLM (+), MdX (-), AMAN (-), MTE (+), GlyA (-), dMAN (-), dMNE (-), dMLZ (-), NAG (-), PLE (-), IRHA (-), BGLU (-), BMAN (-), PHC (-), PVATE (+), AGLU (-), dTAG (-), dTRE $(+)$, INU (-), dGLU (+), dRIB (+), PSCNa (-), NaCl 6.5\% (+), KAN (+), OLD (-), ESC (+), TTZ (-), POLYB_R $(+)$. Detalles bioquímicos de la muestra del agua de fermentación: PyrA (+), APPA (+), ELLM (-). Detalles bioquímicos de la muestra del tanque de fermentación: LeuA (+), PyrA (+), TyrA (+), APPA (+), MTE (-).

En uno de los tanques de fermentación de cemento (4) se encontró Geotrichum capitatum. Las colonias presentaron características macroscópicas (textura cremosa, elevación convexa y borde entero) y microscópicas de levaduras. Hongo levaduriforme patógeno, ambiental cosmopolita. 93\% de probabilidad utilizando la tarjeta YST. Detalles bioquímicos: LysA (+), IMLTa (-), LeuA (+), ARG (+), ERYa (-), GLYLa(+), TyrA (+), BNAG (-), ARBa (-), AMYa (-), dGALa (-), GENa (-), dGLUa (+), LACa (-), MadGa (-), dCELa (-), GGT (-), dMALa (-), dRAFa (-), NAGA1 (-), dMNEa (+), dMELa (-), dMLZa (-), ISBEa (+), IRHAa (-), XLTa (-), dSORa (-), SACa (-), URE (+), AGLU (-), dTURa (-), dTREa (-), NO3a (-), IARA (-), dGATa (+), ESC (-), IGLTa (+), dXYLa (-), LATa (+), ACEa (+), CITa (-), GRTas (+), IPROa (+), 2KGa (+), NAGa (-), dGNTa (+).

En Colombia, la fermentación de almidón de yuca se utiliza para la producción de almidón agrio, llevado a cabo principalmente, en condiciones de anaerobiosis y la cual requiere de una etapa posterior de secado. Sin embargo, hay pocos estudios relacionados con el aislamiento e identificación de microorganimos aerobios procedentes de estos procesos. Sánchez (Sánchez H. et al. 2005), encontraron en muestras de almidón de yuca: 8 colonias del género Bacillus, una de Kurtia y otra de Clostridium, identificadas bioquímicamente; por otro lado, Ampe (2001) reportó Lactobacillus plantarum, Lactobacillus cellobiosus, Leuconostoc mesenteroides, Leuconostoc dextranicum, Weisella paramesenteroides, Pediococcus pentosaceus, Lactococcus lactis, Lactobacillus manihotivorans, Streptococcus salivarius y Lactobacillus casei, identificados molecularmente. Asimismo, Omar (Omar et al. 2000) en otros estudios, encontró las mismas especies de Lactobacillus y adicionalmente, reporta Lactobacillus hilgardii, Lb. buchneri y Lb. fermentum.

En este estudio, también se aislaron microorganismos patógenos como Aeromonas salmonicida, Sphingomonas paucimobilis, Acinetobacter Iwolfii, Crypctococcus laurentii, Geotricum capitatum causando infecciones hospitalarias entre otros; y en particular Bacillus cereus por su constante presencia en las muestras, a pesar de su capacidad amilolítica, genera riesgo de intoxicación diarréica y emética por consumo en alimentos. Los cuales deben ser descartados para futuros procesos de fermentación industrial o extracción de enzimas. Actualmente, los productos de estas modificaciones artesanales son utilizados para la industria de la panadería, por lo que sufren un proceso térmico que es suficiente para evitar efectos negativos que pueden provocar estos patógenos. Sin embargo, esto depende del buen procesamiento en la cocción o de la concentración de estos en el producto final

Por el contrario, Bacillus amiloliquefaciens y Bacillus vallismortis serían buenos candidatos para procesos de almidones modificados, debido a su capacidad de hidrolizar almidón, característica principal en la cual se basó esta investigación y que difiere de otros estudios. Kimaryo (2000) sugiere la inoculación con una o varias especies o cepas de BAL seleccionadas para proporcionar un mejor control y calidad de productos fermentados de almidón de yuca, como el kivunde en Tanzania. Sin embargo, Quintero (2012) sugiere el uso de Aspergillus niger.

Por su alta actividad amilolítica, varias cepas proporcionan azúcares tales como glucosa o maltosa que puede ser utilizado como una fuente de energía por otros microorganismos. Ampe (2001) sugiere que bacterias como $L b$. manihotivorans está presente sólo durante el primer período de fermentación, al degradar el almidón, mientras que $L b$. plantarum se encuentra en todo el proceso y contribuye a la acidificación del producto. Otras investigaciones en la fermentación de la yuca de Brasil, Lacerda (2005) documentó la presencia de los hongos Geotrichum, Galactomyces y Issatchenkia spp.

La presencia de aislamientos patógenos en la producción natural de almidón agrio muestra la importancia de la implementación de BPM (Torres et al. 2010). Además, éstos plantean la necesidad de una producción estandarizada en la que se garantice que estos cultivos bacterianos se encuentran libres de los mismos. Por otro lado, la identificación de bacterias amilolíticas se presenta como antecedente para futuros procesos en que se quiera estudiar el uso de una única cepa o cepas específicas como modificadoras de almidones. 


\section{Presencia de hidrólisis de almidón o actividad amilolítica}

De los microorganismos aislados y purificados, se descartaron las levaduras y 11 aislamientos de bacterias tras encontrar poca o nula actividad amilolítica (menor a $1 \mathrm{mmm}$ de radio). A un total de 36 aislamientos bacterianos diferentes con actividad amilolítica se les midió el radio del halo de hidrólisis de almidón y se reporta la distancia promedio de los halos y el error estándar, además se realizó una comparación múltiple utilizando el procedimiento de diferencia mínima significativa (LSD) DE Fischer con un nivel del 95\% de confianza (Tabla 2) como método de clasificación de un mayor potencial de producción de amilasas. Algunos de estos microorganismos provenían de más de una fuente, por lo que fueron renombradas como FD (fermentación y salida del rallador), FA (fermentación y salida del colador), las otras son nombradas sólo por la fuente de la que provenían; AD (líquido inicial de fermentación), DS (salida del rallo), FC (Fermentación en tanque de cemento).

Tabla 2. Actividad amilolítica en microorganismos aislados del almidón de yuca en una rallandería artesanal.

\begin{tabular}{|c|c|c|c|c|c|c|c|}
\hline Cód. & $\begin{array}{l}\text { D. prom } \pm \text { EE } \\
(\mathrm{mm})\end{array}$ & Cód. & $\begin{array}{l}\text { D. prom } \pm \text { EE } \\
(\mathrm{mm})\end{array}$ & Cód. & $\begin{array}{l}\text { D. prom } \pm \text { EE } \\
(\mathrm{mm})\end{array}$ & Cód. & $\begin{array}{l}\text { D. prom } \pm \text { EE } \\
(\mathrm{mm})\end{array}$ \\
\hline FD1 & $7.3 \pm 0.3^{a}$ & AD2 & $7.0 \pm 0.0^{a b}$ & FD3 & $6.7 \pm 1.2^{\mathrm{abc}}$ & AD4 & $6.7 \pm 0.3^{\mathrm{abc}}$ \\
\hline FC5 & $6.7 \pm 0.6^{\mathrm{abc}}$ & AD6 & $6.7 \pm 0.3^{a b c}$ & AD7 & $6.7 \pm 0.3^{a b c}$ & FC8 & $6.3 \pm 0.3^{a b c d}$ \\
\hline DS9 & $6.3 \pm 0.3^{\mathrm{abcd}}$ & AD10 & $6.3 \pm 0.3^{\mathrm{abcd}}$ & AD11 & $6.3 \pm 0.9^{a b c d}$ & AD12 & $6.3 \pm 0.9^{a b c d}$ \\
\hline AD13 & $6.0 \pm 0.6^{\mathrm{abcde}}$ & AD14 & $6.0 \pm 0.0^{\text {abcde }}$ & AD15 & $6.0 \pm 0.6^{\text {abcde }}$ & FC16 & $5.7 \pm 0.3^{\text {bcdef }}$ \\
\hline AD17 & $5.7 \pm 0.3^{\text {bcdef }}$ & DS18 & $5.7 \pm 0.9^{\text {bcdef }}$ & FA19 & $5.7 \pm 0.9^{\text {bcdef }}$ & FA20 & $5.7 \pm 0.3^{\text {bcdef }}$ \\
\hline DS21 & $5.3 \pm 0.7^{\text {cdef }}$ & FC22 & $5.3 \pm 0.3^{\text {cdef }}$ & AD23 & $5.3 \pm 0.7^{\text {cdef }}$ & AD24 & $5.3 \pm 0.3^{\text {cdef }}$ \\
\hline AD25 & $5.3 \pm 0.3^{\text {cdef }}$ & AD26 & $5.3 \pm 0.9^{\text {cdef }}$ & AD27 & $5.3 \pm 0.3^{\text {cdef }}$ & DS28 & $5.3 \pm 0.7^{\text {cdef }}$ \\
\hline AD29 & $5.3 \pm 0.9^{\text {cdef }}$ & AD30 & $5.0 \pm 0.0^{\text {defg }}$ & AD31 & $5.0 \pm 0.6^{\text {defg }}$ & FC32 & $4.7 \pm 0.3^{\text {efg }}$ \\
\hline AD33 & $4.3 \pm 0.3^{\mathrm{fg}}$ & FC34 & $4.3 \pm 0.3^{\mathrm{fg}}$ & FC35 & $4.3 \pm 0.3^{\mathrm{fg}}$ & AD36 & $3.7 \pm 0.7^{g}$ \\
\hline
\end{tabular}

Los microorganismos con mayores halos de hidrólisis de almidón coinciden con algunos de los identificados como Bacillus amyloliquefaciens (FD1, AD4), Bacillus vallismortis (AD2, FD3, FC5) y Leuconostoc mesenteroides (FC8) son bacterias prometedoras para procesos industriales como la producción de amilasas o la degradación de almidón y producción de dextrinas y etanol, entre otros. También sugiere su uso como bacterias nativas en fermentaciones naturales como la producción de almidón agrio, de la cual se recolectarion las muestras. Además, estas podrían ser parte de procesos de modificación combinado en el que se requiera una etapa de hidrólisis de almidón, debido a la producción de azúcares como glucosa o maltosa que además son fuente energética de otros tipos de microorganismos.

\section{CONCLUSIONES}

En el proceso de producción artesanal de almidón agrio se caracterizaron macro y microscópicamente un total de 76 aislamientos microbianos aerobios. El almidón nativo en este proceso está expuesto a diferentes condiciones ambientales, lo que hace que la obtención de almidón agrio no sea un proceso controlado ni estandarizado. Las condiciones ambientales, así como el tipo de tanque que se utiliza en la fermentación, influyen en la variabilidad de la carga microbiana de cada uno de los tanques y más aún cuando no se presentan condiciones específicas para favorecer o inhibir el crecimiento de ciertos microorganismos de interés.

La identificación de microorganismos demuestra la variedad de microorganismos presentes en las fermentaciones, entre los que se encontraron Bacillus amyloliquefaciens, Bacillus vallismortis, Bacillus cereus, Leuconostoc mesenteroides y Saccharomyces cereviseae. Con base en los microorganismos patógenos encontrados, es clara la necesidad de controlar el proceso, usando microorganismos benéficos o no patógenos que posean actividad amilolítica. Los microorganismos patógenos encontrados como Aeromonas salmonicida, Geotrichum capitatum, Sphingomonas paucimobilis, Acinetobacter Iwolfii, Crytococcus laurentii y Bacillus cereus evidencian la necesidad de la implementación de Buenas Prácticas de Manufactura en la producción de almidones agrios.

Este estudio puede ser utilizado como antecedente para investigaciones de mejoramiento de producción de almidón fermentado, por ejemplo, en los procesos de hidrólisis de almidón, en los que una gran variedad de microorganismos se ven involucrados. Es importante medir la actividad amilolítica de los microorganismos debido a que éstas pueden actuar en conjunto en diferentes etapas fermentativas. Además, medir la actividad amilolítica en aislamientos, que podrían utilizarse para la extracción de amilasas en la hidrólisis del almidón. 


\section{NOTACIÓN}

2KGa: Asimilación Ceto-D-Gluconato

5KG: 5-Ceto-D-Gluconato

ACEa: Asimilación Acetato

ADH1: Arginina Dihidrolasa 1

ADH2s: Arginina Dihidrolasa 2

ADO: Adonitol

AGAL: Alfa-Galactosidasa

AGLU: Alfa-Glucosidasa

AGLTp: Glutamil Arilamidasa Pna

AlaA: Alanina-Arilamidasa

AMAN: Alfa-Manosidasa

AMY: D-Amigdalina

AMYa: Asimilación Amigdalina

APPA: Alanina-Fenilalanina-Prolina-Arilamidasa

ARBa: Asimilación Arbutina

ARG: Arginina GP

AspA: L-Aspartato Arilamidasa

BACI: Resistencia a Bacitrina

BAlap: Beta-Alanina-Amilamidasa-pNA

BGAL: Beta-Galactosidasa

BGAR: Beta-Galatopiranosidasa

BGLU: Beta-Glucosidasa

BGUR: Beta-Glucoronidasa

BMAN: Beta-Manosidasa

BNAG: Beta-N-Acetil-Glucosaminidasa

BXYL:Beta-Xilosidasa

CDEX: Ciclodextrina

CIT: Citrato (sodio)

CITa: Asimilación Citrato (sodio)

CMT: Coumarato

dCEL: D-Celobiosa

dCELa: Asimilación D-Celobiosa

dGAL: D-Galactosa

dGATa: Asimilación D-Galacturonato

dGALa: Asimilación D-Galactosa

dGLU: D-Glucosa

dGLUa: Asimilación D-Glucosa

dGNTa: Asimilación D-Gluconato

dMAL: D-Maltosa

dMALa: Asimilación D-Maltosa

dMAN: D-Manitol

dMELa: Asimilación D-Melibiosa

dMLZ: D-Melesitosa

dMLZa: Asimilación D-Melesitosa

dMNE: D-Manosa

dMNEa: Asimilación D-Manosa

dRAF: D-Rafinosa

dRAFa: Asiilación D- Rafinosa

dRIB: D-Ribosa

dSOR: D-Sorbitol

dSORa: Asimilación D-Sorbitol

dTAG: D-Tagatosa

dTRE: D-Trealosa

dTREa: Asimilación D-Trealosa

dTURa: Asimilación D-Turanosa

dXYL: D-Xilosa

dXYLa: Asimilación D-Xilosa

ELLM: Ellman

ERYa: Asimilación Eritritol

ESC: Hidrólisis Esculina

GENa: Asimilación Gentiobiosa

GGAA: Glutamina-Glicina-Arginina Arilamidasa

GGT: Gamma-Glutamil-Transferasa
GlyA: Glicina-Arllamidasa

GLYG: Glicogeno

GLYLa: Asimilación Glicerol

GRTas: Asimilación Glucuronato

H2S: Producción de H2S

INO: myo-Inositol

INU: Inulina

LAC: Lactosa

LACa: Asimilación Lactosa

IARAa: Asimilación L-Arabinosa

IARL: L-Arabitol

LATa: Asimilación D-Lactato

LDC: Lisina Decarboxilasa

LeuA: Leucina-Arilamidasa

IGLTa: Asimilación L-Glutamato

IHiSa: Asimilación L- Histidina

LIP: Lipasa

ILATa: Asimilación L-Lactato

ILATk: L- Lactato-Alcanilización

IMLTa: Asimilación L-Malato

IPROa: Asimilación L-Prolina

IRHAa: Asimilación Ramnosa

ISBEa: Asimilación L-Sorbosa

KAN: Resistencia Kanamicina

LysA: L-Lisina-Arilamidasa

MagGa: Asimilación Metil-D-Glucopiranosido

MBdG: Metil-B-D-Glucopiranosido

MdX: Metil-D-Xilosida

MTE: Maltotriosa

MNT: Malonato

NAG: N-Acetil-D-Glucosamina

NAGa: Asimilación N-Acetil-Glucosamina

NAGA: Beta-N-Acetil-Galactosamidasa

NAGA1: PNP-N-Acetil-BD- Galactosaminidasa 1

NC 6.5/ $\mathrm{NaCl} 6.5 \%$ : Crecimiento en $6.5 \% \mathrm{NaCl}$

NO3a: Asimilación Nitrato

NOVO: Resistencia a Novobiocina

O129R: O/Resistencia 129

ODC: Ornitina Decarboxilasa

ODEC: Base Decarboxilasa

OFF: Fermentación/Glucosa

OLD: Resistencia Oleandomicina

OPTO: Resistencia a Optochina

PheA: Fenilalanina Arilamidasa

PHC: Fosforil Colina

PHOS: Fosfatasa

PIPLC: Fosfatidilinositol Fosfolipasa C

PLE: Palatinosa

POLYB/ POLYB R: Resistencia Polimixina B

ProA: L-Prolina-Ārylamidasa

PUL: Pululan

PVATE: Piruvato

PSCNa: Asimilación Putrecina

PyrA: L-Pirrolidonil-Arilamidasa

SAC: Sacarosa/Sucrosa

SACa: Asimilación Sacarosa/Sucrosa

SAL: Salicina

SUCT: Succinato-Alcalinización

TyrA: Tirosina-Arilamidasa

TTZ: Tetrazolium Red

URE: Ureasa

XLTa: Asimilación Xilitol 


\section{AGRADECIMIENTOS}

Los autores agradecen a Colciencias, Luz Marina Gómez (Laboratorio de Microbiología de la Fundación Intal) Sebastián Ochoa, Rosalba Alzate y Angela Mora del Laboratorio de Microbiología de Aguas y Alimentos y al Laboratorio de Bioconversiones de la Universidad Nacional de Colombia.

\section{REFERENCIAS}

Acosta, H.A.; Villada, H. S. y Prieto P. A., Envejecimiento de almidones termoplásticos agrios de yuca y nativos de papa por microscopía de fuerza atómica, Información tecnológica. [online]. 2006, vol.17, n.3, pp. 71-78. ISSN 0718-0764. http://dx.doi.org/10.4067/S0718-07642006000300011

Alarcón, M. y Dufuor D. Almidón agrio de yuca en Colombia, en La yuca en el tercer milenio Sistemas modernos de producción, procesamiento, utilización y comercialización, primera edición, CIAT, 470-502, Cali, Colombia (1998)

Ampe, F.; Sirvent, A. y Zakhia, N. Dynamics of the microbial community responsible for traditional sour cassava starch fermentation studied by denaturing gradient gel electrophoresis and quantitative rRNA hybridization. International Journal of Food Microbiology 65 (1-2): 45-54 (2001)

Benesi, I. R. M y otros cuatro autores. The effect of genotype, location and season on cassava starch extraction. Euphytica 160(1): 59-74 (2008)

Benesi, I. R. M.; Labuschagne, M. T.; Dixon, A. G. O. y Mahungu, N. M. Stability of native starch quality parameters, starch extraction and root dry matter of cassava genotypes in different environments. Journal of the Science of Food and Agriculture 84(11): 1381-88 (2004)

BioMérieux, Inc. Vitek 2: Healthcare. Biomérieux. http://www.biomerieux-usa.com/clinical/vitek-2-healthcare. (2015)

Cadena, M. P.; Villarraga, E. C.; Luján, D. E. y Salcedo, J. G. Evaluación de la agroindustria del almidón agrio de yuca (Manihot esculenta Crantz) en Córdoba y Sucre. Temas agrarios 11(1): 43-53 (2006)

Cárdenas, O. S. y Buckle, T. S. Sour cassava starch production: a preliminary study. Journal of Food Science 45(6): 1509-12 (1980)

Cereda, M. P. Microorganismos e ácidos orgânicos ocorrentes na fermentaçao de fécula de mandioca. Anais da academia brasileira de ciências 47: 361-62 (1975)

Figueroa, C.; Davila A. M. y Pourquié J. Lactic acid bacteria of the sour cassava starch fermentation. Letters in Applied Microbiology 21(2): 126-30 (1995)

George, M.; Moorthy, S. N. y Padmaja, G. Biochemical changes in cassava tuber during fermentation and its effect on extracted starch and residue. Journal of the Science of Food and Agriculture 69(3): 367-71. (1995)

Kimaryo, V.M.; Massawe, G.A.; Olasupo, N.A. y Holzapfel, W.H. The use of a starter culture in the fermentation of cassava for the production of 'kivunde', a traditional Tanzanian food product. International Journal of Food Microbiology 56(2-3): 179-90 (2000)

Lacerda, I y otros cinco autores. Lactic acid bacteria and yeasts associated with spontaneous fermentations during the production of sour cassava starch in Brazil. International Journal of Food Microbiology 105(2): 213-19 (2005)

Madigan, Michael T. y otros cinco autores. Brock Biology of Microorganisms. 14th edition. Pearson, 85 - 117 San Francisco, Estados Unidos (2014)

Marcon, M.J.A. y otros cinco autores. The effect of fermentation on cassava starch microstructure. Journal of Food Process Engineering 29(4): 362-72 (2006)

McGrance, S. J.; Cornell H. J. y Rix C. J. A simple and rapid colorimetric method for the determination of amylose in starch products. Starch - Stärke 50(4): 158-63 (1998)

Nunes, O. L. G. S. y Cereda, M. P. Efeito de inóculos na fermentaçao da fécula de mandioca: qualidade organoléptica. En VIII Congresso brasileiro de mandioca (1994)

Olomo, V. y Ajibola, O. Processing factors affecting the yield and physicochemical properties of starch from cassava chips and flour. Starch - Stärke 55(10): 476-81 (2003)

Omar, N. B. y otros cuatro autores. Molecular diversity of lactic acid bacteria from cassava sour starch (Colombia). Systematic and applied microbiology 23(2): 285-91 (2000) 
Parada, J. L.; Zapata E.; Fabrizio, S. V. y Martínez, A. Microbiological and technological aspects of cassavastarch fermentation. World Journal of Microbiology and Biotechnology 12(1): 53-56 (1996)

Quintero C.; Giraldo, V. G. y Lucas J. Modificación del almidón de yuca por A. niger. Vitae 19(1) (2012)

Rojas-Triviño, A. Conceptos y práctica de microbiología. Primera edición. Editorial Universidad Nacional de Colombia, Sede Palmira 13 - 68, Palmira, Colombia (2011)

Sánchez H., Claudia P. y otros seis autores. Estudio de cepas nativas amilolíticas. Vitae, Revista de la facultad de química farmacéutica 12(2): 21-28 (2005)

Torres, Patricia y otros cuatro autores. Una mirada a la agroindustria de extracción de almidón de yuca, desde la estandarización de procesos. Revista Escuela de Ingeniería de Antioquia 14: 23-38 (2010)

Vargas, P.; Araya, Y.; López, R. y Bonilla, A.R. Características de calidad y digestibilidad in vitro del almidón agrio de yuca (Manihot esculenta) producido en Costa Rica. Revista Venezolana de Ciencia y Tecnología de Alimentos 3(1): 1-13 (2012)

Witczak, M.; Ziobro, R.; Juszczak, L. y Korus, J. Starch and starch derivatives in gluten-free systems - A review. Journal of Cereal Science (2015) 